\title{
O USO DE PSICOFÁRMACOS NO MUNICÍPIO DE JOSÉ DA PENHA/RN E SUAS IMPLICAÇÕES PARA A SAÚDE MENTAL
}

\author{
Werena de Oliveira Barbosa ${ }^{1}$; Bertulino José de Souza² \\ ${ }^{1}$ Mestranda do Programa de Pós-Graduação em Planejamento e Dinâmicas \\ Territoriais no Semiárido - PLANDITES, da Universidade do Estado do Rio Grande \\ do Norte, Pau dos Ferros, Rio Grande do Norte, Brasil. werenabarbosa@gmail.com \\ 2 Professor Doutor do Programa de Pós-Graduação em Planejamento e Dinâmicas \\ Territoriais no Semiárido - PLANDITES, da Universidade do Estado do Rio Grande \\ do Norte, Pau dos Ferros, Rio Grande do Norte, Brasil.

\section{Recebido em: 15/11/2021 - Aprovado em: 15/12/2021 - Publicado em: 30/12/2021 \\ DOI: 10.18677/EnciBio_2021D33} \\ trabalho licenciado sob licença Creative Commons Attribution-NonCommercial-NoDerivatives 4.0 International License.
}

\section{RESUMO}

O uso de psicofármacos vem aumentando consideravelmente, devido aos novos diagnósticos de transtornos psiquiátricos antes desconhecidos, do desenvolvimento da indústria farmacêutica, o aparecimento de novos fármacos no mercado e das novas indicações terapêuticas. Essas substâncias são mais conhecidas como antidepressivos e tranquilizantes, e, na realidade, são medicamentos com ações alucinógenas que agem no sistema nervoso central, causando alterações de conduta, humor, cognição e estado mental. O presente estudo dedicou-se a compreender na perspectiva dos usuários de saúde mental no município de José da Penha-RN, os significados e as necessidades do uso de psicofármacos. Nesse sentido, a pesquisa aprovada por Comitê de Ética em pesquisa investigou as origens da demanda, o processo de concessão/prescrição dos medicamentos e uma hipotética dependência do uso. A metodologia utilizada foi de caráter qualitativo, exploratório e descritivo com aplicação de questionário semiestruturado com indivíduos maiores de 18 anos e usuários de psicofármacos. A análise dos dados cotizada pela Análise do Discurso permitiu compreender parte do expresso pelos depoentes. Os resultados apontaram para a necessidade de se promover um maior conhecimento sobre as necessidades de ações e planejamento que auxiliem na utilização adequada desses medicamentos.

PALAVRAS-CHAVE: Atenção Básica, Psicofármacos. Saúde Mental.

\section{THE USE OF PSYCHOPHARMACEUTICALS IN JOSÉ DA PENHA/RN CITY AND ITS IMPLICATIONS FOR MENTAL HEALTH}

\begin{abstract}
The use of psychotropic drugs has been increasing considerably, due to new diagnoses of previously unknown psychiatric disorders, the development of the pharmaceutical industry, the appearance of new drugs on the market and new therapeutic indications. These substances are better known as antidepressants and tranquilizers, and, in fact, they are medications with hallucinogenic actions that act on the central nervous system, causing changes in behavior, mood, cognition and mental state. This study aimed to understand, from the perspective of mental health users in the city of José da Penha-RN, the meanings and needs of the use of
\end{abstract}


psychotropic drugs. In this sense, the research approved by the Research Ethics Committee investigated the origins of demand, the process of granting/prescribing drugs and a hypothetical dependence on use. The methodology used was qualitative, exploratory and descriptive, with application of a semi-structured questionnaire with individuals over 18 years of age and psychotropic drug users. Data analysis quoted by Discourse Analysis allowed us to understand part of what was expressed by the deponents. The results pointed to the need to promote greater knowledge about the needs for actions and planning that help in the proper use of these drugs.

KEYWORDS: Psychopharmaceuticals. Mental Health. Primary Care.

\section{INTRODUÇÃO}

Os psicofármacos são conhecidos como antidepressivos, tranquilizantes e medicamentos com ações alucinógenas que agem no sistema nervoso central, causando alterações de conduta, humor, cognição e o estado mental (MARTINS, 2003). Dados apontados pela Organização Mundial de Saúde (OMS) apresentam um aumento no consumo dessas substâncias, devido aos novos diagnósticos de transtornos psiquiátricos antes desconhecidos, do desenvolvimento da indústria farmacêutica, o aparecimento de novos fármacos no mercado e das novas indicações terapêuticas (PRADO et al., 2017).

A introdução desses medicamentos na terapêutica permite uma atuação mais rápida, diminuindo as perturbações mentais, enquanto outras terapias de custos mais baixos, como a psicoterapia e a atividade física possuem efeitos menos adversos, porém, trazem um resultado não tão imediato (LOYOLA FILHO et al., 2014). O seu uso vem sendo cada vez mais investigado através de pesquisas de base populacional, justificando-se pela facilidade de acesso da população aos medicamentos a partir de sua crescente utilização, pela possibilidade de assinalar o uso não racional, e pela importância em restituir o potencial funcional dos usuários após a estabilização clínica da doença (PRADO et al., 2017).

A associação de psicofármacos está relacionada a busca de uma substância para alívio de algo que incomoda ou de uma determinada doença/dor/sofrimento, e isso pode refletir na maximização ou minimização de efeitos relacionados a percepção de sensações, bem como na disponibilidade de medicamentos e os padrões de consumo próprios de momentos ou contextos específicos (AZEVEDO; OLIVEIRA, 2011).

De acordo com a OMS, avalia-se que cerca de 700 milhões de pessoas sofrem de doenças mentais, uma vez que esses transtornos afetam uma em cada 10 pessoas em todo o mundo, e apresentam 13\% do total de enfermidades do planeta (SANTOS et al., 2018). As doenças mentais se classificam como neurológicas e psiquiátricas, e para sua diferenciação, Macedo et al., (2018) justificam que, de acordo com a agência americana Food and Drug Administration (FDA), responsável pela aprovação de novos fármacos e alimentos, na área de neurologia, estão os fármacos para as doenças de Alzheimer e Parkinson, por exemplo. $\mathrm{Na}$ área de psiquiatria encontram-se patologias como depressão, esquizofrenia, transtorno bipolar e transtorno do pânico, entre outras.

Para Alves et al., (2020), a maioria da população com prevalência dessas doenças, especialmente os portadores de Transtornos Mentais Comuns (TMC), são atendidos na Atenção Primária à Saúde (APS). Tais transtornos abrangem sintomas leves como insônia, fadiga, irritabilidade, esquecimento, dificuldade de concentração e queixas somáticas, os quais, isoladamente não preenchem os critérios formais para o diagnóstico de depressão e/ou ansiedade, não sendo 
tratados com sua total eficácia nos serviços da APS, o que pode fazer com que os pacientes frequentem os vários serviços de saúde em busca de respostas para seus sintomas.

No Brasil, os transtornos mentais podem constituir até um terço da demanda na Atenção Básica $(A B)$, chegando a $50 \%$, quando se consideram os quadros subsindrômicos, os quais possuem características semelhantes a uma síndrome, como os TMCs, contribuindo para o aumento de prescrições, especialmente dos antidepressivos e dos benzodiazepínicos, mesmo essas substâncias apresentando efeitos adversos, podendo causar dependência e problemas à saúde se usados por períodos prolongados, o seu consumo tem representado uma parcela significativa do total de medicamentos prescritos (ALVES et al., 2020).

Sarmento e Santos (2019) afirmam que no Brasil, o Ministério da Saúde instituiu, em 2007, o Comitê Nacional para a Promoção do Uso Racional de Medicamentos, que prevê a desprescrição e a desmedicamentalização, objetivando promover a diminuição e o uso racional de medicamentos no país, enfatizando que a disponibilização de medicamentos deve ocorrer de acordo com as necessidades clínicas dos usuários e nas doses e períodos apropriados, considerando seu custo e a relação risco-benefício. Em relatório técnico da Organização das Nações Unidas (ONU), sobre a produção de psicofármacos, em 2017, o Brasil é apontado como o quarto maior produtor mundial de benzodiazepínicos, segundo maior consumidor de fenobarbital e sétimo maior importador de metilfenidato (SARMENTO; SANTOS, 2019).

Ao tratar-se das questões de saúde/doença mental no âmbito da Atenção Primária à Saúde (APS), o uso de psicofármacos só deve ser estabelecido dentro de um contexto de escuta e formação de vínculo entre profissionais de saúde e usuário (BRASIL, 2013). A partir dessa conexão, o usuário deverá compreender que o uso da medicação passará a implicar não só na troca de receitas como também em um processo de cuidado, diante das queixas que os fazem buscar o serviço de atendimento nas Unidades Básicas de Saúde (UBSs).

O uso de diversas medicações por um mesmo indivíduo, leva a um aumento da incidência de interações medicamentosas e potencializa o risco de uso inadequado (REIS et al., 2018). Como esses medicamentos devem ser utilizados de forma racional, devido ao seu potencial de abuso, o desenvolvimento da dependência, o surgimento de efeitos colaterais e a sua prescrição é regulada pelo Ministério da Saúde (MS) (ALVES et al., 2020).

De acordo com Reis et al., (2018), a ocorrência de renovação de receitas sem uma avaliação clínica é frequente nas UBSs, o que permite a existência de rotinas próprias de renovação incorporadas à prática cotidiana de serviços da Atenção Básica $(A B)$, abrindo espaço para questionamentos a respeito dos benefícios que traz à população. Esse contexto costuma gerar uma demanda básica, que nem sempre consegue se organizar de forma adequada para reavaliar corretamente prescrições, por vezes múltiplas, fornecidas aos usuários (REIS et al., 2018).

O tema em questão representa o interesse em entender sociologicamente a maneira que, através de programas e políticas sociais, poderão ser elaboradas estratégias para a manutenção ou superação de problemas relacionados à saúde mental na Atenção Básica (AB). O estudo pretendeu mostrar, em suas múltiplas manifestações socio espaciais, especialmente no âmbito da saúde mental, as relações sociais que implicam no desenvolvimento de uma política pública de saúde eficaz. 
O encontro das observações in loco com a bibliografia utilizada exprimiu, enquanto suposição desse estudo, que o município em questão apresenta um grande fluxo de pacientes de saúde mental que fazem uso de psicofármacos, sendo necessário um melhor planejamento de critérios que possam atende-lo, como a padronização na forma de prescrição de receitas e no fortalecimento da relação de vínculo entre profissionais de saúde e usuários, para uma possível minimização desses danos.

Portanto, teve como objetivo, compreender na perspectiva dos usuários de saúde mental no município de José da Penha-RN, os significados e as necessidades do uso de psicofármacos. Também, i) identificar as principais categorias de usuários que fazem uso de psicofármacos, quanto a necessidade do uso da medicação; ii) realizar um levantamento dos psicofármacos mais utilizados pela população do município; iii) dialogar entre diferentes teorias e conceitos relacionados à saúde mental, no âmbito da Atenção Básica; iv) discutir a respeito da disponibilização de medicamentos no município de acordo com as necessidades clínicas dos usuários; v) descrever como esses mecanismos são compreendidos pelos usuários de saúde mental, por meio de suas narrativas.

Desse modo, o foco principal do estudo consistiu no uso de psicofármacos e,

nesse sentido, investigou as origens da demanda, 0 processo de concessão/prescrição dos medicamentos e de uma hipotética dependência, aqui ponderada. Sob essas condições, buscou-se capturar o significado da necessidade do uso dos psicofármacos e as suas representações, a partir das subjetividades dos usuários, sintonizado ao trabalho dos profissionais de saúde no âmbito da Atenção Básica $(A B)$, bem como às instituições que os atendem.

\section{MATERIAIS E MÉTODOS}

Frente aos acontecimentos referentes à pandemia ocasionada pela Covid-19, os procedimentos metodológicos deste estudo foram orientados pela Carta Circular no 1/2021-CONEP/SECNS/MS de 3 de março de 2021, que trata de procedimentos com pesquisas virtuais em tempos de pandemia (BRASIL, 2021).

Para o alcance dos objetivos, constituiu-se um trabalho de caráter exploratório e descritivo. Segundo Piovesan e Temporini (1995), a pesquisa exploratória apresenta uma essência qualitativa e contextual, utilizada quando se pretende realizar investigações sobre fatores humanos, sendo necessário elaborar um instrumento baseado nas experiências reais dos sujeitos, na sua linguagem e ambiente de vida, a fim de se obter um estudo prévio da realidade, que deve estar sempre presente na fase de planejamento. Quanto à pesquisa descritiva ela pretende narrar os fatos e fenômenos de determinada realidade exigindo do investigador uma série de informações sobre o que deseja pesquisar (SILVEIRA; CÓRDOVA, 2009).

Para o melhor entendimento destes elementos, utilizou-se a pesquisa quantitativa, salientando as experiências subjetivas dos usuários. Compreende-se que ela apresenta relação dinâmica entre mundo objetivo e subjetividade do sujeito, que não pode ser traduzida em números. É voltada para a descoberta, identificação, descrição aprofundada e geração de explicações, além de buscar o significado e a intencionalidade dos atos contemplando o propósito deste trabalho (DUARTE, 2002).

A pesquisa foi realizada no município de José da Penha $\left(6^{\circ} 19^{\prime} 6^{\prime \prime}\right.$ sul, $38^{\circ} 16^{\prime}$ 44" oeste), localizado no interior do estado do Rio Grande do Norte, região Nordeste 
do país. Situa-se na microrregião de Pau dos Ferros e mesorregião do Oeste Potiguar, distando 421 quilômetros a oeste da capital do estado, Natal. De acordo com o Instituto Brasileiro de Geografia e Estatística (IBGE, 2021), ele ocupa uma área de aproximadamente $118 \mathrm{~km}^{2}$ e sua população, no censo de 2010, era de 5.868 habitantes, sendo então o $101^{\circ}$ (centésimo primeiro) município mais populoso do estado e 6으 (sexto) de sua microrregião.

Diante da abrangência da pesquisa, o público-alvo foi formado por meio das 3 (três) Unidades Básicas de Saúde (UBSs) existentes no município de José da Penha, que fazem atendimento aos usuários de saúde mental: o Centro de Saúde Francisca Rodrigues Fernandes, sediada no centro da cidade; a UBS Maria do Socorro Nunes, localizada na zona rural Vila Major Felipe; e a UBS da Ema, situada na zona rural Carnaubinha/Ema. Dessa forma, o objetivo foi alcançar, no mínimo, $20 \%$ (vinte por cento) do total de usuários de saúde mental de cada Unidade Básica de Saúde. Compreendeu-se que este quantitativo é suficiente para a pesquisa, no qual as questões particulares aos sujeitos não podem ou não devem ser quantificadas (MINAYO, 2012).

A amostra foi composta pelos usuários de saúde mental que fazem uso de psicofármacos disponibilizados pela rede de Atenção Básica do município de José da Penha. A descrição das características dos participantes não foi priorizada por não haver diferenciação por idade, sexo, raça/cor, etnia, orientação sexual, identidade de gênero, classes ou grupos sociais e grupos vulneráveis, a tônica foi a utilização do psicofármacos.

A seleção dos participantes se deu a partir dos seguintes critérios: i) idade superior a 18 anos; ii) estar cadastrado no sistema de Prontuário Eletrônico Cidadão (PEC); iii) fazer uso de psicofármacos atualmente. Foram incluídos na população amostral apenas usuários que concordaram em participar, voluntariamente, da pesquisa. Excluíram-se menores de 18 anos e pessoas que não utilizavam psicofármacos. Como instrumento de pesquisa foi utilizado um questionário com entrevista semiestruturada, assegurada a impessoalidade e o sigilo de todas as informações prestadas, segundo a Resolução 466/12 do Conselho Nacional de Saúde.

A coleta de dados foi mediada via ambiente virtual, a qual envolveu a utilização de formulário disponibilizado e do uso de aplicativo online, assim, como consta nas orientações da Carta Circular no 1/2021, na qual, devido às restrições vivenciadas pela população mediante a pandemia da Covid-19, o contato não envolveria a presença física do pesquisador e do participante da pesquisa. Entretanto, devido as circunstâncias e entraves encontradas no percurso metodológico, tornou-se necessário a presença da pesquisadora em alguns momentos para orientar o preenchimento do formulário online, que ocorreu via aplicativo de WhatsApp, através do link para endereço eletrônico https://forms.gle/i1HkBXaaFVZf54b36.

Para análise de dados, utilizaram-se os pressupostos teóricos de uma corrente de reflexão denominada Análise de Discurso. Logo, o entendimento do discurso foi visto como "um conjunto de signos que expressaria um pensamento, ou algo que distorceria a realidade ou mesmo que faria as coisas 'falarem' - trazer à tona seus significados ocultos - por meio da palavra" (FERREIRA; TRASVERSINI, 2013, p. 209, grifos dos autores).

Para Orlandi (2005), a Análise do Discurso tem a noção de funcionamento como central, levando o analista a compreendê-lo pela observação dos processos e mecanismos de constituição de sentidos e de sujeito, lançando mão da paráfrase e 
da metáfora como elementos que permitem um certo grau de operacionalização dos conceitos.

A análise dos dados consistiu, ainda, em ponderar os resultados através dos discursos e relatos de usuários referentes ao uso de psicofármacos, junto a bibliografia existente, a partir dos quais identificou-se os conceitos relativos à linguagem, sociedade e aos processos sociais. Dessa forma, procurou-se observar o que Gonçalves (2016), descreve como uma relação entre língua e ideologia, uma vez que, a análise do discurso atua com o sentido e não com o conteúdo do texto, um sentido que não é traduzido, mas produzido, bastando que sua materialidade produza sentidos para interpretação, permitindo enxergar além do que está escrito, nas entrelinhas, como os estereótipos, os estigmas e as vertentes políticaideológicas.

Quanto ao referencial teórico, buscou-se abordar questões a respeito do que é Saúde Mental, da criação da Política Nacional de Saúde Mental (PNSM), bem como das reflexões profissionais dentro da Atenção Básica, além de enfatizar os conceitos sobre psicofármacos.

\section{RESULTADOS E DISCUSSÕES}

Inicialmente, foi realizado o acesso ao sistema de Prontuário Eletrônico Cidadão (PEC), o qual deveria constar dados e registros para que fossem identificados os pacientes que fazem uso de medicamentos relacionados a saúde mental. No entanto, constatou-se que o sistema PEC possui uma grande deficiência de informações, não sendo possível identificar os participantes através desse método. Percebeu-se, com isso, a extrema importância da função do Agente Comunitário de Saúde (ACS), o qual deve alimentar esse sistema com todas as informações possíveis sobre os usuários, inclusive sobre o uso de medicamentos psicofármacos, o que não acontece na prática.

Dessa forma e diante do cenário pouco promissor da coleta, optou-se por utilizar uma estratégia alternativa, mas conectada com a demanda da pesquisa: agregar 15 (quinze) Agentes Comunitários de Saúde (ACSs) do município e solicitar que cada um deles indicasse 5 (cinco) pessoas de sua área que apresentassem uma demanda de saúde mental e fizessem o uso de psicofármacos, para que os mesmos pudessem responder o formulário de perguntas, totalizando um número final de 75 (setenta e cinco) participantes. Diante da dificuldade de alguns participantes em utilizar tecnologias eletrônicas e aplicativos de celular, foi necessária a presença da primeira pesquisadora para auxiliar no preenchimento do formulário on line.

A coleta de dados foi realizada no segundo semestre de 2021 e, entre reuniões com os ACSs e preenchimento dos formulários online, a duração da pesquisa de campo foi de 20 dias. Após a coleta de dados, realizou-se uma organização dos materiais, através de leitura, análises e registro dos relatos. Mediante os resultados realizou-se a estratificação de ideias e conceitos, a fim de relacionar com o referencial teórico que trata sobre a temática.

Os dados coletados serão apresentados, a seguir, através dos quais foram fornecidas as informações de acordo com a compreensão dos usuários de psicofármacos atendidos nas UBSs do município. As respostas referentes a pesquisa realizada foram fornecidas por 75 sujeitos, os quais concordaram livremente em participar da pesquisa.

Diante dos resultados encontrados, percebeu-se a prevalência dos participantes com o uso de medicamentos psicofármacos nos últimos 3 dias que 
antecederam a entrevista. Os medicamentos indicados no Quadro 1 foram utilizados pelos participantes desta pesquisa e ilustram a diversidade e os grupos farmacológicos existentes no universo do estudo.

QUADRO 1 - Distribuição dos principais medicamentos psicofármacos utilizados pelos sujeitos da pesquisa em grupos farmacológicos no município de José da Penha-RN, no ano de 2021

\begin{tabular}{|c|c|c|c|c|}
\hline $\begin{array}{l}\text { Medicamento } \\
\text { Psicofármaco }\end{array}$ & $\begin{array}{l}\text { Ansiolíticos } \\
\text { e Hipnóticos }\end{array}$ & $\begin{array}{l}\text { Antidepre } \\
\text { ssivos }\end{array}$ & $\begin{array}{c}\text { Antipsicóticos } \\
\text { ou } \\
\text { neurolépticos }\end{array}$ & $\begin{array}{c}\text { Estabilizadores } \\
\text { de } \\
\text { humor }\end{array}$ \\
\hline
\end{tabular}

$\begin{array}{ll}\text { Clonazepam } & \mathrm{x} \\ \text { Alprazolam } & \mathrm{x} \\ \text { Diazepam } & \mathrm{x} \\ \text { Dalmadorm } & \mathrm{x} \\ \text { Zolpidem } & \mathrm{x}\end{array}$

Amitriptilina $\quad x$

Nortriptilina $\quad x$

Fluoxetina $\quad x$

Sertralina $\quad x$

Paroxetina $\quad x$

Citalopram $\quad x$

Escitalopram $\quad x$

Venlafaxina $\quad x$

Bupropiona $\quad x$

Trazodona $\mathrm{x}$

Risperidona

$\mathrm{X}$

Quetiapina $\quad X$

Melleril $\quad X$

Neozine $\quad x$

Prebictal $\quad \mathrm{X}$

Akineton $\quad \mathrm{X}$

Carbamazepina $x$

Topiramato $x$

Ácido Valpróico $\quad x$

Gardenal $\quad$ x

Divalproato de $\quad x$

Sódio

Fonte: Elaborado pelos autores, (2021).

Sabe-se, que os psicofármacos são medicamentos que se dividem em classes como antidepressivos, antiepiléticos, ansiolíticos, antipsicóticos e estabilizadores do humor e são designados para tratamento de transtornos mentais. Os usuários de psicofármacos geralmente apresentam problemas relacionados à ansiedade, depressão, insônia, psicoses maníacas, esquizofrenia e outros sintomas (CANCELLA, 2012).

Quando perguntado sobre o uso de bebidas alcoólicas, tendo-a na condição de um dos potencializadores dos quadros de saúde, sobretudo pela liberalidade social do consumo, $92 \%$ dos participantes responderam que não fazem uso, enquanto $8 \%$ afirmaram consumir bebidas alcóolicas. De acordo com Belloti et al., (2017), o Levantamento Nacional de Álcool e Drogas (LENAD) aponta que 17\% dos 
brasileiros são dependentes do álcool ou usam de forma abusiva - o causador de 1,8 milhões de mortes por ano no mundo, acometendo em sua maioria jovens entre $15 \mathrm{e}$ 29 anos. Dessa forma, cerca de 60 patologias estão relacionadas ao uso prejudicial do álcool (DUARTE et al., 2018). A cultura nacional e internacional demonstra que, desde cedo, é ensinado que tomar uma cerveja com os amigos é um ato social, visto como sinônimo de diversão e distração, apesar de todos os riscos que isso acarreta.

Tendo em vista que droga é toda substância que, ao ser introduzida, inalada, ingerida ou injetada, provoca alterações no funcionamento do organismo, modificando suas funções, como é abordado por Cavalcante et al., (2008), o álcool classifica-se como uma droga psicoativa, que se mostra relevante na discussão também pelo seu uso indiscriminado, abordando questões referentes à vulnerabilidade dos indivíduos no plano individual e social. $O$ uso e o abuso de álcool entre outras drogas compõem as principais causas desencadeadoras de situações de vulnerabilidade social, através de acidentes, suicídios, violência, gravidez indesejada e a transmissão de doenças por via sexual ou injetável (CAVALCANTE et al., 2008).

Conforme Teixeira (2006), o álcool é uma droga subestimada, pois na cultura atual é encarada como fonte integrante de uma vida normal, que reflete todos os ambientes e situações possíveis, associando-se a desportos, viagens, trabalho, entre outros. Por ser permitida a venda e consumo desse produto, torna-se importante problematizar seu uso, as patologias que desencadeia e o uso de substâncias que a combatam.

Wannmacher (2007), aponta que a maioria dos estudos sobre interações medicamentosas com álcool considera pacientes que fazem uso crônico e abusivo de bebidas alcoólicas, entretanto, há menos informação sobre aqueles que bebem moderadamente, uma vez que geralmente para se discutir sobre esse assunto interessa saber se seu uso é em pequena quantidade ou em sobrecarga aguda (ocasional) ou característico do alcoolismo (crônico e sistemático). No entanto, um estudo italiano multicêntrico associou o uso moderado de álcool ao aumento de $24 \%$ no risco de reações adversas a fármacos (ONDER et al., 2002).

Uma vez que o alcoolismo representa um estigma social, pacientes dependentes de bebida alcóolica tendem a omitir essa informação, o que pode levar profissionais de saúde a prescreverem medicamentos com potencial de interação, acarretando efeitos indesejáveis. O questionário Cut down, Annoyed by criticism, Guilty e Eye-opener (CAGE), por exemplo, é um instrumento de anamnese que permitem maior esclarecimento, a fim de que o médico se oriente com relação ao diagnóstico do problema e prescrição de medicamentos destinados a tratamento de comorbidades. (WANNMACHER, 2007).

Outra pesquisa indica que, entre 869 pacientes adultos atendidos na Atenção Primária à Saúde (APS), os quais fazem uso de bebidas alcóolicas e que recebem medicamentos, $40 \%$ estavam tomando medicamentos com potencial de interação com álcool, mais frequentemente bupropiona e compostos contendo paracetamol. (BROWN et al., 2007).

Para Oga et al., (2002), a interação medicamentosa é a consequência de uma intervenção no efeito de uma droga por outra droga, comidas, bebidas ou até mesmo por alguns agentes químicos ambientais. Além disso, algumas medicações possuem uma grande capacidade de desempenhar interação de forma adversa com bebidas alcóolicas, uma vez que essas drogas causam alteração no metabolismo do álcool, resultando no aumento ou diminuição dos níveis sanguíneos. Paulo (2006), corrobora que o álcool pode intervir na eficácia de alguns medicamentos, 
acentuando os seus efeitos e alterando o metabolismo dos fármacos, afetando também as concentrações destas substâncias no corpo.

A respeito do uso concomitante dos benzodiazepínicos e bebidas alcoólicas, a interação dessas duas substâncias pode provocar uma intoxicação grave, uma vez que o álcool aumenta a taxa de absorção destes fármacos e a depressão do sistema nervoso central a eles associada, o que pode levar a alterações nas funções psíquicas e à diminuição da atividade cardiovascular e respiratória (SÃO PAÚLO, 2012; GOODMAN; GILMAN, 2012). Desse modo, Silva (2017), complementa que os efeitos ansiolíticos dessas drogas são amplamente aumentados pelo álcool e a ingestão de ambos tende a aumentar o efeito sedativo do fármaco podendo levar uma pessoa a insuficiência respiratória e ao risco de coma ou morte.

Também foi questionado aos participantes sobre o uso de cigarro, os quais $5,4 \%$ disseram ser fumantes, $13 \%$ responderam que são ex-fumantes e $81,6 \%$ nunca fumaram. A nicotina é a principal responsável por inúmeras interações na farmacodinâmica de alguns fármacos como, por exemplo, os benzodiazepínicos, além disso, também pode interferir na farmacocinética, por inibição do metabolismo hepático enzimático, ou por alterações na excreção de alguns fármacos (ANTONIO, 2013).

Considerando que a prescrição de psicofármacos é frequente como coadjuvante no tratamento da dependência ao tabaco, existem medicamentos específicos para esses casos. Desse modo, observa-se que existe uma relação entre tabagismo e saúde mental, como mostra um estudo realizado em 2003. (RODRIGUES et al., 2006). Outro estudo realizado em São Paulo indicou maior prevalência de Transtornos Mentais Comuns (TMCs) em fumantes quando comparados a não fumantes. (VIDAL et al., 2013).

Dessa forma, é reconhecida, hoje, a importância do acesso às concepções populares sobre essa realidade para que se possa pensar e elaborar medidas preventivas e de controle. Assim, buscam-se novas formas de compreensão que aprofundem às representações e comportamentos associados ao uso de psicofármacos para que se possa intervir nessas situações.

Nesse sentido e à guisa de conhecer as ações dos sujeitos, indagou-se aos participantes sobre a prática da atividade física $55,2 \%$ afirmaram não praticar nenhuma atividade, enquanto $44,8 \%$ responderam que sim. Dentre as principais práticas destacaram-se: caminhada, treino funcional e ciclismo.

A literatura aponta que a prática de atividades físicas é uma terapia que pode ser usada em associação aos psicofármacos para o tratamento dos transtornos depressivos, bem como a psicoterapia cognitivo comportamental, que tem sido um método bem sucedido para prevenção e reincidência da psicopatologia, com isso, o tratamento com base nessas terapias integrativas agrega a recuperação ao paciente (TOMÉ; VALENTINI, 2006). Diante disso, pondera-se que no mundo contemporâneo, a forma como as pessoas vivem, se alimentam, se relacionam, suas escolhas, habilidades ou não de viver socialmente, não ficam apenas no contexto externo, elas são simbolizadas pela mente e pelo sentir. E é a interpretação do que vivem e sentem que diz um pouco sobre a sua saúde mental.

Com as novas tecnologias do mundo moderno, a era da internet e as constantes exigências quanto ao trabalho e as obrigações diárias, os momentos de lazer têm se tornado escassos, gerando níveis de estresse que podem acarretar transtornos mentais. 
Talvez uma parte considerável dos distúrbios observados no mundo moderno seja decorrente de sintomas relacionados ao estresse, como a ansiedade e a agressividade. Para auxiliar no tratamento e prevenção desses distúrbios a prática de atividades físicas pode ser considerada uma alternativa. Programas sistemáticos de atividades físicas são mais efetivos para produzir mudanças positivas na sensação de bem-estar do que programas ocasionais, benefícios que são observados em diferentes idades. (TOMÉ, VALENTINI, 2006, p. 129).

Para Araújo et al., (2006), a não realização de atividades físicas cotidianamente pode interferir na capacidade de resistência cardiorrespiratória, o que normalmente resulta em complicações na realização de tarefas cotidianas relacionadas à vida profissional e de lazer, aumentando as chances que o indivíduo tem de desenvolver doenças como osteoporose, hipertensão, doenças coronarianas, diabetes, como também ansiedade, depressão e alguns estados negativos do humor. Isso se dá pelo fato de que a prática regular de exercícios físicos aeróbios pode produzir efeitos antidepressivos e ansiolíticos e proteger o organismo dos efeitos prejudiciais do estresse na saúde física e mental.

A prática de exercícios físicos tem apresentado resultados promissores em termos de saúde pública, principalmente no âmbito das reações emocionais a situações estressoras de medo que podem desencadear os transtornos de ansiedade. É importante destacar que é na interação de mecanismos fisiológicos e psicológicos que o bem-estar psicológico expressado pelo praticante de atividade física se otimiza, com a redução dos níveis de ansiedade e agressividade (TOMÉ, VALENTINI, 2006). Outro aspecto relacionado ao bem-estar questionado aos participantes foi quanto ao tempo diário de sono, os quais $35,1 \%$ responderam menos de 6 horas e 64,9\% disseram 6 horas ou mais. Quando questionado aos participantes a respeito do que achavam sobre os serviços de saúde do município, obtiveram-se as seguintes falas:

"Para mim os serviços de saúde estão deixando muito a desejar, médico nos postos tem todos os dias agora quando a pessoa precisa de algum exame que coloca na prefeitura não sai." (entrevistado 9).

"Considero, nos dias atuais regular. Na farmácia popular deveria haver remédios controlados, em virtude que são muito caros e todos os que eu uso eu compro e não tenho emprego." (entrevistado 12).

"É fraco." (entrevistado 31).

"É devagar. Aguardo consulta desde agosto do ano passado..." (entrevistado 44).

Quando questionados se possuíam alguma doença crônica 47,3\% disseram que não, enquanto 52,7\% afirmaram que sim. Dentre as falas, as principais doenças crônicas encontradas foram: Hipertensão, Diabetes, Osteoporose, Cardiopatia, Cirrose, Epilepsia, Câncer, Enxaqueca, Ansiedade e Depressão.

De acordo com Vidal et al., (2013), em pesquisa sobre Transtornos Mentais Comuns (TMCs) em mulheres, apontam que as doenças clínicas mais relatadas são hipertensão arterial, diabetes e problemas osteomusculares, as quais demonstram uma associação entre a presença de doenças clínicas e a ocorrência de TMCs, uma ENCICLOPÉDIA BIOSFERA, Centro Científico Conhecer - Jandaia-GO, v.18 n.38; p. 188 2021 
vez que doenças físicas crônicas graves e incapacitantes têm sido correlacionadas frequentemente com a presença de sintomas psiquiátricos.

A expressão Transtorno Mental Comum (TMC), criada por Goldberg e Huxley, compreende sintomas depressivos e ansiosos como insônia, fadiga, irritabilidade, esquecimento, dificuldade de concentração e queixas somáticas. Esses sintomas, mesmo quando acompanhados por incapacidade funcional e sofrimento, não preenchem os critérios formais para diagnósticos de depressão e/ou ansiedade (VIDAL et al., 2013, p. 458).

Tal semelhança pode ser observada em um estudo multicêntrico, no qual indivíduos vivendo com uma doença orgânica crônica têm maior probabilidade de receber uma prescrição de psicofármaco em razão de irem à consulta médica com maior frequência. Com isso, depreende-se que existe a associação entre diagnóstico médico de hipertensão e consumo de psicofármacos (RODRIGUES et al., 2006).

Ainda quando questionado se os participantes consideram ter algum problema emocional, $52,63 \%$ disseram que sim, enquanto $47,37 \%$ responderam que não. Com relação aos participantes apresentarem distúrbio de insônia, 60,53\% afirmaram que sim, $26,31 \%$ disseram que não e $13,16 \%$ relataram que às vezes sim.

Os problemas emocionais, assim como os transtornos mentais têm alcançado uma prevalência mundial e nacional. Na Atenção Básica tem se mostrado muito relevante, chegando a um terço da demanda, taxas essas que alcançam e até ultrapassam os $50 \%$ quando se inclui o sofrimento difuso com sintomas psiquiátricos subsindrômicos. Transtornos mentais são frequentes na população, principalmente entre os pacientes atendidos nos serviços de saúde, sendo mais prevalentes entre o sexo feminino, nos indivíduos com pouca escolaridade, baixa renda, tabagistas e nas mulheres vítimas de violência. (VIDAL et al., 2013).

No entanto, tais transtornos são pouco identificados ou tratados e tendem a ser subestimados pelos profissionais de saúde, principalmente quando sintomas físicos não estão presentes. As ações da Estratégia de Saúde da Família (ESF) poderiam favorecer a detecção desses transtornos e o encaminhamento dos pacientes para tratamento, se consideradas suas características de acessibilidade e cobertura (OMS, 2001; RIBEIRO et al., 2009; SÃO PAULO, 2011).

A ausência de Centros de Atenção Psicossocial (CAPS) no município de José da Penha também contribui para o alto índice de transtornos em saúde mental, isso se deve ao fato de o município apresentar um número de 5.868 habitantes (de acordo com o último censo de 2010), não se enquadrando no valor demográfico suficiente para receber esse tipo de serviço. Sua maior população se concentra na zona rural, porém, a atuação profissional junto aos usuários de saúde mental torna-se necessária nas três UBSs existentes no município. De acordo com - Ministério da Saúde (MS), a proposta de reorganizar o Programa de Saúde da Família (PSF) e as Unidades Básicas de Saúde (UBSs) devem servir para estabelecer vínculos de compromisso e responsabilidade entre os profissionais de saúde e a população, cuja premissa é a porta de entrada para qualquer forma de adoecimento e atendimento à saúde (CORREIA et al., 2011).Dessa forma, quando questionados se procuraram por serviço/profissional de saúde nos últimos 15 dias, $11 \%$ dos participantes responderam que sim, enquanto $89 \%$ disseram que não. 
Questionou-se também, a respeito se sofreram alguma internação durante esse ano. A esse respeito $9 \%$ dos participantes responderam que sim, enquanto 91\% disseram que não. Os participantes ainda relataram os seguintes motivos:

"Tive que me consultar porque minha diabetes estava descompensada" (entrevistado 23).

"Esses dias baixei no hospital com crise de ansiedade" (entrevistado $69)$.

E quando questionado se possuem algum plano de saúde $100 \%$ dos participantes afirmaram não possuir nenhum plano.

Como forma de se alcançar um dos objetivos dessa pesquisa, realizou-se um levantamento dos principais psicofármacos dispensados pela Farmácia Básica do município no período de um mês, entre setembro e outubro de 2021 (tabela 1).

TABELA 1 - Medicamentos Psicofármacos dispensados pela Farmácia Básica. José da Penha, 2021.

\begin{tabular}{|c|c|c|c|c|}
\hline $\begin{array}{l}\text { Medicamento } \\
\text { Psicofármaco }\end{array}$ & $\begin{array}{l}\text { Dose por } \\
\text { Unidade } \\
\text { Posológica } \\
\text { (mg) }\end{array}$ & $\begin{array}{l}\text { № de } \\
\text { Usuários }\end{array}$ & $\begin{array}{c}\text { Quantidade } \\
\text { de } \\
\text { Comprimidos } \\
\text { Dispensados }\end{array}$ & $\begin{array}{l}\text { Média de } \\
\text { comprimidos por } \\
\text { usuário* }\end{array}$ \\
\hline Clonazepam & $2 \mathrm{mg}$ & 36 & 2.340 & 65 \\
\hline Bromazepam & $3 \mathrm{mg}$ & 7 & 420 & 60 \\
\hline $\begin{array}{l}\text { Carbonato de } \\
\text { lítio }\end{array}$ & $300 \mathrm{mg}$ & 5 & 300 & 60 \\
\hline Alprazolam & $1 \mathrm{mg}$ & 4 & 210 & 52,5 \\
\hline Amitriptilina & $25 \mathrm{mg}$ & 26 & 1.120 & 43,08 \\
\hline Paroxetina & $20 \mathrm{mg}$ & 7 & 300 & 42,86 \\
\hline Fluoxetina & $20 \mathrm{mg}$ & 22 & 930 & 42,27 \\
\hline Bromazepam & $6 \mathrm{mg}$ & 5 & 210 & 42 \\
\hline Sertralina & $50 \mathrm{mg}$ & 13 & 532 & 40,92 \\
\hline Sertralina & $100 \mathrm{mg}$ & 3 & 120 & 40 \\
\hline Escitalopram & $20 \mathrm{mg}$ & 31 & 1230 & 39,68 \\
\hline Diazepam & $10 \mathrm{mg}$ & 8 & 300 & 37,5 \\
\hline Clonazepam & $0,5 \mathrm{mg}$ & 9 & 330 & 36,67 \\
\hline Nortriptilna & $50 \mathrm{mg}$ & 5 & 180 & 36 \\
\hline Alprazolam & $0,5 \mathrm{mg}$ & 6 & 210 & 35 \\
\hline Citalopram & $20 \mathrm{mg}$ & 59 & 2.010 & 34,07 \\
\hline Amitriptilina & $75 \mathrm{mg}$ & 6 & 180 & 30 \\
\hline
\end{tabular}

* Quantidade variável de acordo com a posologia indicada na receita.

Fonte: Elaborada pelos autores, (2021).

Diante dos números alcançados observa-se a quantidade de medicamentos distribuídos gratuitamente e quantidade de pessoas que fazem uso dessas substâncias num curto período de um mês. Dessa forma, torna-se imprescindível o debate e a relevância de um planejamento que envolva todas a Instâncias responsáveis pela saúde pública. Trata-se aqui do bem-estar de seres humanos, que necessitam de uma atenção direcionada ao seu problema específico.

O tratamento dos transtornos mentais e/ou de comportamento, quando necessário se usar um psicofármaco, é preciso ponderar se a relação risco-benefício ENCICLOPÉDIA BIOSFERA, Centro Científico Conhecer - Jandaia-GO, v.18 n.38; p. 190 
potencial da droga justifica seu emprego e se outros recursos foram devidamente explorados, para que não se possa prescrever qualquer substância, as quais muitas vezes são desnecessárias. Por isso, no tratamento psicofarmacológico é de suma importância avaliar os sintomas e não buscar objetivos gerais, mas específicos, de acordo com o sintoma-alvo, uma vez que, ao pertencer a quadros sindrômicos ou categoriais distintos, receberá indicações de tratamento e/ou de psicofármacos de grupos totalmente diferentes (BRASIL, 2000).

Por exemplo, num paciente o sintoma de "hiperatividade psicomotora" pode estar presente no transtorno de déficit de atenção/hiperatividade (TDAH), num transtorno difuso do desenvolvimento, no retardo mental, em transtornos do humor, em transtornos de ansiedade e em situações relativas ao ambiente. (BRASIL, 2000, p. 40).

Nessa perspectiva, Rodrigues, et al., (2006), apresentam em seu estudo uma forte associação entre consulta médica e o consumo de psicofármacos, na qual a consulta médica torna-se um momento crucial para que médicos e pacientes possam ter um entendimento entre ambos, de forma que a prescrição de um psicofármaco seja o resultado de uma indicação adequada.

Entre os aspectos apresentados, destaca-se a importância de ações relacionadas ao rastreamento, acompanhamento e encaminhamento de casos que necessitam de atendimento em saúde mental. Tais ações demandam, no entanto, investimento em capacitação contínua das equipes de ESF para o alcance desse objetivo. Por fim, quanto ao elevado número de pessoas, sem indícios de transtorno mental, fazendo uso de medicamentos psicofármacos merece uma maior atenção e reflexão, evidentemente com intervenções terapêuticas não restritas à prescrição de medicamentos.

\section{CONCLUSÕES}

A utilização indiscriminada de psicofármacos ocorre em diversos contextos geográficos, espaços culturais, classes sociais e em diferentes faixas etárias, provocando grandes impactos pessoais e sociais, além de alto custo econômico. Desse modo, constitui um dos problemas de saúde pública, de transcendência social e, em face disso, requer políticas de controle e prevenção dessa prática, que envolvam vários setores da sociedade como saúde, educação e assistência social.

Essa problemática social encontrada no município de José da Penha/RN expressa uma grande preocupação com a saúde dos usuários de psicofármacos que necessitam de um acompanhamento direcionado as suas demandas de forma mais específica. Diante desta concepção, as UBSs são a porta de entrada para o atendimento a pacientes que apresentam diagnósticos de comprometimento da saúde mental, e, enquanto cidadãos, necessitam de atenção e qualidade de vida saudável para o seu desenvolvimento individual e coletivo.

Na pesquisa realizada observou que os serviços oferecidos para a população com demandas de saúde mental são extremamente escassos e insuficientes, tanto pelo aparato técnico-operativo quanto pelos recursos humanos, pois, o município necessita melhorar os mecanismos de atendimento e acompanhamento dos casos para esses usuários.

Da mesma forma, ao se analisar os dados qualitativos dessa pesquisa, mais especificamente os que se direcionam a intervenção direta aos usuários de 
psicofármacos, percebeu-se a precariedade das ações desenvolvidas. Estão relacionadas a esse contexto à desresponsabilização dos entes governamentais e gestores em ampliar as ações e medidas que possam a promover a diminuição no uso de psicofármacos. Logo, a sociedade civil se encontra inebriada pelo apelo do consumismo e individualismo social presente na contemporaneidade, revelando a ampliação da desregulamentação e precarização dos serviços.

\section{REFERÊNCIAS}

ALVES, E. O.; VIEIRA, P. D. A.; OLIVEIRA, R. A. S.; RODRIGUES, R. F.; SILVA, S. C. et al. Prevalência do uso de psicotrópicos na atenção primária à saúde em um município do interior de Minas Gerais. Revista Médica de Minas Gerais, [S.I.], v. 30, (Sulp 4), p. 61-68, 2020. Disponível em: http://rmmg.org/artigo/detalhes/2710. Acesso em: 10 fev. 2021.

ANTÓNIO, M. G. Interações do tabagismo com a terapêutica farmacológica. 2013. 61f. Dissertação (Mestrado Integrado em Ciências Farmacêuticas) - Instituto Superior de Ciências da Saúde Egas Moniz, 2013. Disponível em: https://comum.rcaap.pt/bitstream/10400.26/14164/1/Ant\%c3\%b3nio\%2c\%20Mara\%2 0Guedes.pdf. Acesso em: 23 ago. 2021.

ARAÚJO, S. R. C.; MELLO, M. T.; LEITE, J. R. Transtornos de ansiedade e exercício físico. Brazilian Journal of Psychiatry, [S.I.], v. 29, p. 164-171, 2007. Disponível em:

https://www.scielo.br/j/rbp/a/fLNhMjCxYWyzQLDYWFYNBCs/?format=pdf\&lang=pt. Acesso em: 3 out. 2021.

AZEVEDO, R. C.; OLIVEIRA, K. D. Poliusuários de substâncias psicoativas. In: Dependência química: prevenção tratamento e políticas públicas. $1^{\underline{a}}$ ed, São Paulo: Artmed, 2011.

BELLOTI, M.; FRAGA, H. L.; BELOTTI, L. Family and psychosocial attention: care for the person with abusive use of alcohol and other drugs. Cadernos Brasileiros de Terapia Ocupacional. [S.I.], v. 25, n. 3, p. 617-625, 2017.

BRASIL, H. H. A. Princípios gerais do emprego de psicofármacos. Revista Brasileira de Psiquiatria, [S.I.], v. 22, (Supl II), p. 40-41, 2000. Disponível em: https://www.scielo.br/j/rbp/a/FSnWzWxtbnqcbyswTNwgkdP/?lang=pt\&format=pdf.

Acesso em: 12 set. 2021.

BRASIL. Ministério da Saúde. Secretaria de Atenção à Saúde. Departamento de Atenção Básica. Saúde mental / Ministério da Saúde, Secretaria de Atenção à Saúde, Departamento de Atenção Básica, Departamento de Ações Programáticas Estratégicas, Brasília, 2013. 176 p. Disponível em: http://bvsms.saude.gov.br/bvs/publicacoes/cadernos_atencao_basica_34_saude_me ntal.pdf. Acesso em: 10 fev. 2021.

BRASIL. Carta Circular no 1/2021-CONEP/SECNS/MS de 3 de março de 2021. Brasília: DF, Ministério da Saúde, 2021. Disponível em: http://conselho.saude.gov.br/images/comissoes/conep/documentos/CARTAS/Carta_ Circular_01.2021.pdf. Acesso em: 18 abr. 2021. 
BROWN, R. L.; DIMOND, A. R.; HULISZ, D.; SAUNDERS, L. A.; BOBULA, J. A. Pharmacoepidemiology of potential alcohol-prescription drug interactions among primary care patients with alcohol-use disorders. Journal of the American Pharmacists Association, [S.I.], v. 47, n. 2, p. 135-139, 2007. Disponível em: https://www.sciencedirect.com/science/article/abs/pii/S1544319115314035. Acesso em: 5 out. 2021.

CANCELLA, D. C. B. Análise do uso de psicofármacos na Atenção primária: uma revisão de literatura. Trabalho de Conclusão de Curso (TCC). Conselheiro Lafaiete - $\quad$ MG, $2012 . \quad$ Disponível em: https://www.nescon.medicina.ufmg.br/biblioteca/imagem/3400.pdf. Acesso em: 16 jun. 2021.

CAVAlCANTE, M. B. P. T.; ALVES, M. D. S.; BARROSO, M. G. T. Adolescência, álcool e drogas: uma revisão na perspectiva da promoção da saúde. In: Escola Anna Nery Revista de Enfermagem. Rio de Janeiro, v. 12, n. 3, p. 555-559, 2008. Disponível em: http://www.scielo.br/pdf/ean/v12n3/v12n3a24.pdf. Acesso em: 10 mar. 2021.

CORREIA, V. R; BARROS, S.; COLVERO, L. A. Saúde mental na atenção básica: prática da equipe de saúde da família. Revista Escola de Enfermagem, São Paulo, v. 45, n. 6, 2011. Disponível em: https://www.scielo.br/pdf/reeusp/v45n6/v45n6a32.pdf. Acesso em: 14 fev. 2021.

DUARTE, M. P. C.; LINS, S. L. F.; SILVA, G. W. S.; SENA, R. C. F.; ISOLDI, D. M. R. et al. Controle do uso abusivo do álcool com psicofármacos. Revista de Enfermagem, UFPE on line, Recife, v. 12, n. 3, p. 790-800, mar., 2018. Disponível em: https://periodicos.ufpe.br/revistas/revistaenfermagem/article/view/23043/28048. Acesso em: 12 out. 2021.

DUARTE, R. Pesquisa qualitativa: reflexões sobre o trabalho de campo. Cadernos de Pesquisa, São Paulo, v. 115, p. 139-154, 2002. Disponível em: https://www.scielo.br/j/cp/a/PmPzwqMxQsvQwH5bkrhrDKm/?lang=pt. Acesso em: 15 fev. 2021.

FERREIRA, M. S.; TRAVERSINI, C. S. A Análise Foucaultiana do Discurso como Ferramenta Metodológica de Pesquisa. Educação \& Realidade, Porto Alegre, v. 38, n. 1 , p. 207-226, jan./mar. 2013. Disponível em: https://www.scielo.br/pdf/edreal/v38n1/12.pdf. Acesso em: 30 mar. 2021.

GONÇALVES, A. T. P. Análise de conteúdo, análise do discurso e análise de conversação: estudo preliminar sobre diferenças conceituais e Teóricometodológicas. Administração: Ensino e Pesquisa, Rio de Janeiro, v. 17 n. 2, p. 275-300, 2016. Disponível em: https://raep.emnuvens.com.br/raep/article/view/323/pdf_1. Acesso em: 20 abr. 2021.

GOODMAN, L. S.; GILMAN, A. As Bases Farmacológicas da Terapêutica. 12. ed. Rio de Janeiro: AMGH, 2012. 
IBGE - Instituto Brasileiro de Geografia e Estatistica. População de José da Penha/RN, 2021. Disponível em: https://cidades.ibge.gov.br/brasil/rn/jose-dapenha/panorama. Acesso em: 14 fev. 2021.

LOYOLA FILHO, A. I.; CASTRO-COSTA, E.; FIRMO, J. O. A.; PEIXOTO, S. V. Tendências no uso de antidepressivos entre idosos mais velhos: Projeto Bambuí. Revista Saúde Pública, [S.I.], v. 48, n. 6, dez. 2014. Disponível em: https://www.scielo.br/pdf/rsp/v48n6/pt_0034-8910-rsp-48-6-0857.pdf. Acesso em: 11 abr. 2021.

MACEDO, J. B.; PEREK, K. V.; KARAS, L. P.; PEREIRA, A. V. Fármacos inovadores em saúde mental: uma avaliação das duas últimas décadas. Revista de Medicina, [S. I.], v. 97, n. 4, p. 385-395, 2018. DOI: 10.11606/issn.1679-9836.v97i4p385-395. Disponível em: https://www.revistas.usp.br/revistadc/article/view/142833. Acesso em: 20 abr. 2021.

MARTINS, F. Psicopatologia II. Semiologia psicanalítica: O sintoma simbólico. Brasília: Editora Universidade de Brasília, 2003.

MINAYO, M. C. S. Pesquisa social: teoria, método e criatividade. 32. ed. Petrópolis, Rio de Janeiro: vozes, 2012.

OGA, S.; BASILE, A. C.; CARVALHO, M. F. Guia Zanini-Oga de interações medicamentosas: base teórica das interações. São Paulo: Atheneu, 2002.

ONDER, G.; LANDI, F.; DELLA VEDOVA, C.; ATKINSON, H.; PEDONE, C. et al. Moderate alcohol consumption and adverse reactions among older adults. Pharmacoepidemiol Drug Safety, [S.I.], v. 11, p. 385-392, 2002. Disponível em: https://onlinelibrary.wiley.com/doi/10.1002/pds.721. Acesso em: 6 set. 2021.

OMS - Organização Mundial de Saúde. Relatório sobre a saúde no mundo 2001. Saúde mental: nova concepção, nova esperança. Genebra: OMS; 2001 (tradução para o português pelo Escritório Central OPAS e revisão técnica pela Área Técnica da saúde Mental do Ministério da Saúde do Brasil).

ORLANDI, E. P. Análise do discurso: princípios e procedimentos. 8 ed. Campinas. Pontes: 2005.

PAULO, G. A. Álcool x medicamentos: uma guerra sem vencedores. 2006.

Disponível em: https://www.artwine.com.br/edicoes/wine-style-8-alcool-xmedicamentos-uma-guerra-sem-vencedores.pdf. Acesso em: 6 set. 2021.

PIOVESAN, A. TEMPORINI, E. R. Pesquisa exploratória: procedimento metodológico para estudos de fatores humanos no campo da saúde pública. Revista Saúde Pública, [S.I.], ed. 29, p. 318-25, 1995. Disponível em: https://www.scielo.br/pdf/rsp/v29n4/10.pdf. Acesso em: 11 abr. 2021.

PRADO, M. A. M. B.; FRANCISCO, P. M. S. B.; BARROS, M. B. A. Uso de medicamentos psicotrópicos em adultos e idosos residentes em Campinas, São Paulo: um estudo transversal de base populacional. Revista Epidemiologia e 
Serviços de Saúde, Brasília, v. 26, n. 4 p. 747-758, out/dez 2017. Disponível em: https://www.scielo.br/pdf/ress/v26n4/2237-9622-ress-26-04-00747.pdf. Acesso em: $11 \mathrm{abr} .2021$.

REIS, I. L. F.; ALVES, L. F. C.; CUNHA, L. D. R.; CAVALLI, M. A. P.; AGUIAR, R. A. T. Renovação de Prescrição Médica na atenção primária: uma análise crítica. Revista Médica de Minas Gerais, [S.I.], v. 28, e-1936, 2018. Disponível em: http://rmmg.org/artigo/detalhes/2360. Acesso em: 10 fev. 2021.

RIBEIRO, W. S.; ANDREOLI, S. B.; FERRI, C. P.; PRINCE, M.; MARI, J. J. Exposição à violência e problemas de saúde mental em países em desenvolvimento: uma revisão da literatura. Brazilian Journal of Psychiatry, v. 31, (Suppl 2), p. S49S57, $2009 . \quad$ Disponível em: https://www.scielo.br/j/rbp/a/NXwXSNY6XrmvJ57gzHwst5c/?format=pdf\&lang=pt. Acesso em: 30 out. 2021.

RODRIGUES, M. A. P.; FACCHINI, L. A.; LIMA, M. S. Modificações nos padrões de consumo de psicofármacos em localidade do Sul do Brasil. Revista de Saúde Pública, [S.I.], v. 40, p. 107-114, 2006. Disponível em: https://www.scielo.br/j/rsp/a/J587qqXqDR7vqq8Lcqn5BBw/?format=pdf\&lang=pt.

Acesso em: 19 set. 2021.

SÃO PAULO. Álcool x medicamentos. São Paulo, Conselho Regional de Farmácia de São Paulo (CRF-SP), 2012. Disponível em: http://portal.crfsp.org.br/index.php/noticias/3622-alcool-x-medicamentos.html Acesso em: 21 set. 2021.

SÃO PAULO. Transtornos Mentais Comuns: Tabagismo. Consumo de Bebida Alcoólica. São Paulo: Secretaria Municipal da Saúde. Coordenação de Epidemiologia e Informação - CEInfo, Boletim ISA, 2011, 64 p. Disponível em: https://www.prefeitura.sp.gov.br/cidade/secretarias/upload/saude/arquivos/publicacoe s/Boletim_ISA_5.pdf. Acesso em: 2 out. 2021.

SANTOS, H. S.; NESTOR, A. G. S.; ABREU, B. S.; MODESTO, K. R. A utilização dos medicamentos psicotrópicos e seus fatores associados. Revista de Iniciação Cientifica e Extensão, [S.I.], v. 1, n. 1, p. 51-56, jan./jun. 2018. Disponível em: https://revistasfacesa.senaaires.com.br/index.php/iniciacaocientifica/article/view/48/15. Acesso em: 15 fev. 2021.

SARMENTO, G. A.; SANTOS, S. D. Perspectiva do usuário sobre o acompanhamento e o uso de psicotrópicos na atenção básica. Essentia, Sobral, v. 20, n. $2, \quad$ p. $52-60, \quad 2019$ Disponível em: https://essentia.uvanet.br/index.php/ESSENTIA/article/view/281. Acesso em: $10 \mathrm{fev}$. 2021.

SILVA, S. V. L. A interação do álcool com medicamentos e seus efeitos no organismo. 2017. 27f. (Trabalho de Conclusão de Curso) - Curso de Farmácia, Faculdade de Educação e Meio Ambiente, Ariquemes, 2017. Disponível em: https://repositorio.faema.edu.br/bitstream/123456789/1255/1/SILVA\%2c\%20S.\%20V. $\% 20 L . \% 20-$ 
\%20A\%20INTERA\%c3\%87\%c3\%830\%20DO\%20\%c3\%81LCOOL\%20COM\%20ME DICAMENTOS\%20E\%20SEUS\%20EFEITOS\%20NO\%20ORGANISMO.pdf. Acesso em: 26 jul. 2021.

SILVEIRA, D. T.; CÓRDOVA, F. P. A pesquisa científica. In: Gerhardt, T. E.; Silveira, D. T. Métodos de Pesquisa. Porto Alegre: UFRGS, 2009.

TEIXEIRA, J. M. S. Consumos de álcool na adolescência: início, percursos e prevenção. 2006. 72f. (Trabalho de Conclusão de Curso) - Universidade Fernando Pessoa, Faculdade de Ciências da Saúde/Escola Superior de Saúde, Porto, 2006. Disponível em: https://bdigital.ufp.pt/bitstream/10284/1459/2/Mono_11592.pdf. Acesso em: 17 set. 2021.

TOMÉ, T. H.; VALENTINI, N. C. Benefícios da atividade física sistemática em parâmetros psicológicos do praticante: um estudo sobre ansiedade e agressividade. Journal of Physical Education, [S.I.], v. 17, n. 2, p. 123-130, 2006. Disponível

em: https://periodicos.uem.br/ojs/index.php/RevEducFis/article/view/3350/2407. Acesso em: 2 out. 2021.

VIDAL, C. E. L.; YAÑEZ, B. F. P.; CHAVES, C. V. S.; YAÑEZ, C. F. P.; MICHALAROS, I. A. et al. Transtornos mentais comuns e uso de psicofármacos em mulheres. Cadernos Saúde Coletiva, [S.I.], v. 21, p. 457-464, 2013. Disponível em: https://www.scielo.br/j/cadsc/a/FnkLHMdSfyJmWh5mZL9dXyC/?lang=pt\&format=pdf. Acesso em: 12 set. 2021.

WANNMACHER, L. Interações de medicamentos com álcool: verdades e mitos. Brasília: Organização Pan-Americana da Saúde/Organização Mundial da Saúde, [S.I.], v. 4, n. 12, p. 1-6, 2007. Disponível em: http://www.ufrgs.br/boletimcimrs/2007-

Intera\%C3\%A7\%C3\%B5esmedicamentosx\%C3\%A1lcool.pdf. Acesso em: 2 out. 2021. 